\title{
Ciliary dysfunction secondary to COVID-19. Explanation of the pathogenesis from analysis of human interactome with SARS- CoV-2 proteome
}

\author{
MD Alejandro Jesús Bermejo-Valdés¹, MD Alexander Ariel Padrón-González², \\ MD Jessica Archer Jiménez ${ }^{3}$

\begin{abstract}
${ }^{1}$ Riojan Health Service, Emergency Service 061, Piqueras 98, 26006, Logroño, La Rioja, Spain ${ }^{2}$ Central Laboratory of Cerebrospinal Fluid (LABCEL), Ramón Pinto No. 202, Luyanó, Diez de Octubre, Havana Medical Sciences University, AP 10049, CP 11000, Havana, Cuba

${ }^{3}$ Riojan Health Service, Emergency Service 061, Piqueras 98, 26006, Logroño, La Rioja, Spain
\end{abstract}

\section{Abstract}

Coronavirus Disease-2019 (COVID-19) is an infectious disease caused by the Severe Acute Respiratory Syndrome Coronavirus 2 (SARS-CoV-2). There is sufficient experimental evidence to confirm that SARS-CoV-2 infection produces states of ciliary and flagellar dysfunction. However, these studies are unable to explain the observed effects molecularly, because they lack a sufficient understanding of the interaction between human proteins and virus proteins. Using the physical-chemical study of the human interactome in interaction with the SARS-CoV-2 proteome, we found evidence of interactions to explain the experimental effects from a molecular perspective. We found that ten viral proteins interact with key components in the maintenance of the molecular structure of axoneme. Additionally, we evaluated the pulmonary and extrapulmonary pathogenesis of COVID-19 from the point of view of ciliary dysfunction, and warned about other possible complications such as episodes of transient infertility that, due to the limitations of our work, would need verification.

Key words: COVID-19, SARS-CoV-2, interactome, ciliary-flagellar dysfunction, infertility, pathogenicity

\section{Introduction}

Coronavirus Disease 2019 (COVID-19) was first reported in December 2019 in Wuhan, China. It is an infectious disease caused by Severe Acute Respiratory Syndrome Coronavirus 2 (SARS-CoV-2). Angiotensin Converting Enzyme 2 (ACE2) is a cellular receptor for SARS-CoV-2, and the viral receptor-binding 
protein is spike (S, SARS-CoV-2 S). Although, intervention of CD147 receptor mediated by Cyclophilin-A has also been described, involving the $\mathrm{N}$ protein of SARS-CoV-2 (N, SARS-CoV- $2 \mathrm{~N})^{[1]}$.

COVID-19 is mainly characterized by lung damage ${ }^{[1]}$ but there are collateral clinical manifestations that are not yet well characterized from a pathophysiological point of view, for example: loss of taste or smell.

In United States A, a clinical study described that smell and taste impairments were strongly associated with COVID-19[2] and Centers for Disease Control and Prevention added 'loss of taste or smell' as one of six new symptoms of COVID19 on their advisory page on April 27, 2020[3].

In the human nasal epithelium, coronavirus infects the ciliated cells and causes deciliation ${ }^{[4]}$. But, there is not sufficient molecular evidence to explain these phenomena. However, we begin to see light on this path. A recent study that revealed interaction of viral nonstructural protein 13 (Nsp13) with human centrosome components ${ }^{[4]}$.

The infection of the ciliated cells led us to carry out an active search for possible molecular explanations of deciliation problems, not only to explain the loss of smell and taste. We believe that alteration of ciliary structure can have more devastating consequences of those that are reported. So, we found sufficient molecular interaction between SARS-CoV-2 proteome and human proteome to explain at a general level what is happening in COVID-19, adding that cells susceptible to ciliary (and flagellar) damage express the ACE2 and CD147 receptors that allow SARS-CoV-2 to enter the host cell.

\section{Cilia and mucociliary dysfunction}

Cilia play important physiological functions in human body, and ciliary dyskinesia is responsible for several diseases, generically called ciliopathies ${ }^{[5]}$. Ciliary dyskinesia is associated with recurrent respiratory infections and fertility defects $^{[5]}$.

Mucociliary clearance is an important innate primary defence mechanism and protects the lungs from the deleterious effects of inhaled pollutants, allergens, 
and pathogens ${ }^{[6]}$. The mucociliary system consists of three functional compartments, namely the cilia, a protective mucus layer, and a fluid layer on the surface of the airways, which work together to remove inhaled particles from the lung ${ }^{[6]}$.

\section{Alterations of the ciliary and flagellar structure in lung diseases and male infertility}

Primary ciliary dyskinesia is a genetic disease with symptoms caused by the malfunctioning of motile cilia, such as chronic nasal discharge, complicated respiratory infections, bronchiectasis, and in many cases, male infertility[7]. The genes whose involvement causes primary ciliary dyskinesia have a role in the biogenesis, structure and function of motile cilia[7].

In Drosophila, the hemingway gene ( $\mathrm{hmw}$ ) has been identified as a necessary component for motile cilia function. This gene encodes a protein HMW characterized by a highly conserved coiled-coil domains that is also found in the gene products of their human ortholog, KIAA1430[5] (also known as CFAP97 ${ }^{[8]}$ and in mice ortholog Cfap97d1 (Cfap97 domain containing 1) ${ }^{[9]}$. Cfap97 and Cfap97d1 gene products in mice contain the coiled-coil domains that we mentioned in the study on Drosophila ${ }^{[5,9]}$.

HMW is conserved in species with motile cilia and, in Drosophila, hmw is expressed in ciliated sensory neurons and spermatozoa ${ }^{[5]}$, thus we can be expected that CFAP97 be also expressed in human ciliated sensory neurons and spermatozoa.

Soulavie et al. ${ }^{[5]}$ created hmw-knockout flies and found that it produced poor hearing and sterile males. hmw is involved in the motility of ciliated auditory sensory neurons and, in testes, is necessary for the lengthening and maintenance of sperm flagella[5]. $\mathrm{HMW}$ is not a structural component of the motile axoneme but it is necessary for the adequate acquisition of motile properties ${ }^{[5]}$. In Soulavie et al. study, HMW was identified as a novel component, evolutionarily conserved, necessary for the function of motile cilium and assembly of flagella[5]. Therefore, CFAP97, the human ortholog of hmw, must then have similar functions. 
Soulavie et al. study is about a Drosophila model and it can have drawbacks derived from species difference. Zur Lage et al.. ${ }^{[10]}$ clarified this. They stated that Drosophila study is extensible to humans, and laid the groundwork for increasing use of Drosophila as an excellent model for discovery and validation of a new motile ciliary gene ${ }^{[10]}$. This is necessary to understand the function and assembly of motile cilia, as well as to understand the nature of the genetic defects underlying human motile ciliopathies ${ }^{[10]}$. According to this, we can expect that CFAP97 to be expressed in ciliated sensory neurons and human spermatozoa.

\section{CFAP97 gene family and male infertility}

The flagellum is essential for motility of spermatozoa in vivo. The axonemal is the main component of flagella and spans its entire length ${ }^{[9]}$. An axonemal is composed of 2 central microtubules surrounded by 9 doublets (denoted $9+2$ in scientific literature), the nexin-dynein regulatory complex, the radial rays, and the dynein arms ${ }^{[9]}$. Failure to mount correctly axonemal components in a sperm flagellum results in impaired fertility ${ }^{[9]}$. (Fig.1)

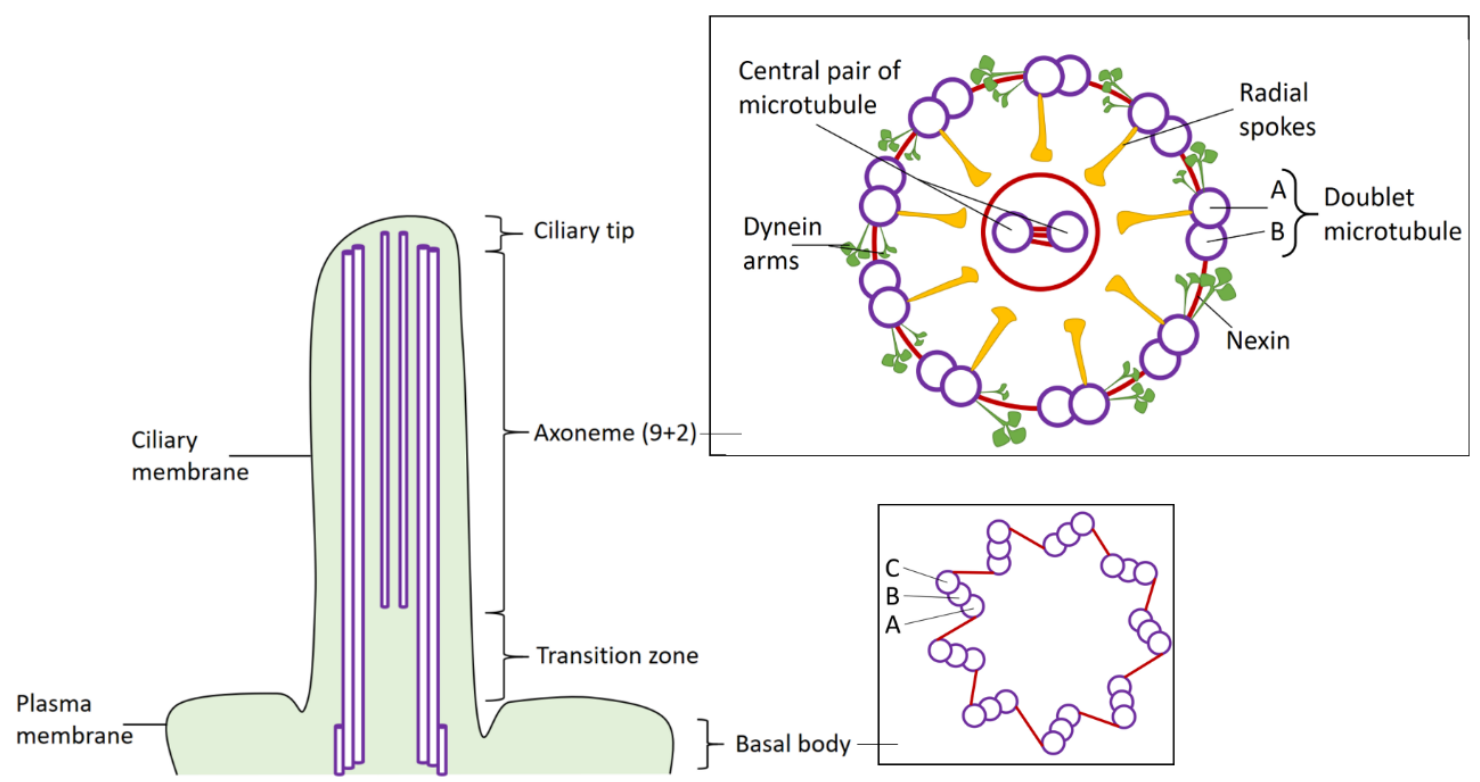

Fig.1. Schematic representation of the most important components of ciliary structure. Left, a longitudinal section diagram of a cilium showing different parts: ciliary tip, axoneme 9+2, transition zone, basal body, plasma membrane and ciliary membrane. Right and up, a cross section diagram of cilium in the part 9+2 axoneme, where we indicate the arrangement of some protein components in that part of cilium: central pair of microtubule, dynein arms, nexin, radial spokes and doublet microtubule. Right and down, cross-sectional diagram of the basal body potion of the ciliary structure, where 
can we see that there is a different microtubule disposition structure (triplet microtubule $\mathrm{ABC})$.

Oura et al. ${ }^{[9]}$ have defined the function of Cfap97d1, an uncharacterized gene in mice. Cfap97d1 is evolutionarily conserved in mammals and many other species, was exclusively expressed in the testes from postnatal day 20 , and continues into adulthood. Deletion of this gene led to sperm motility defects (asthenozoospermia) and male subfertility. Oura et al. study demonstrated that Cfap97d1 is necessary for the maintenance of ultrastructure of the sperm flagellum ${ }^{[9]}$.

Cfap97d1 belongs to family of genes associated with cilia and flagella 97, which contains three members: Cfap97, Cfap97d1 and Cfap97d $2^{[9]}$. Human orthologues exist for all three members ${ }^{[9]}$.

It appears that Cfap97 and Cfap97d1 plays important roles in the maintenance of the ciliary and flagellar structure and, possibly, the function of the product of these genes depends on the coiled-coil domains.

Human and mouse CFAP97 is an ortholog of Chlamydomonas reinhardtii FAP97, whose gene product has been identified in protein extracts from dismembered axonemes $^{[9]}$. It has also been possible to identify the gene product of CFAP97 in a complex with human centrosome-cilium interface proteins ${ }^{[9]}$. In this way, the participation of Cfap97 protein in the human ciliary structure is even clearer.

\section{ACE2 and CD147 expression in testes. Infertility and COVID-19}

SARS-CoV-2 could have an increased affinity by human testicular tissue via ACE2. Therefore, the long-term consequences on fertility in this type of infection should be studied ${ }^{[11]}$. It has been shown that CD147 is also expressed in human testes $^{[12]}$, so that CD147 adds a new possibility of testicular infection. In both cases, via ACE2 ${ }^{[11]}$ or CD147 $7^{[12]}$ germ cells are involved and this should alert us. Knockout of CD147 leads to subfertility in female mice while the male mice are infertile, but the subfertility in female mice was proposed to be resulted from fertilization and implantation failure due to reduced matrix metalloproteinases production $^{[12]}$. 
Taking into account the critical functions of testicular cells for transmission of genetic information, Shen et al. ${ }^{[11]}$ analysed single-cell RNA sequencing data from adult human testes. ACE2 mRNA expression was expressed in both germ cells and somatic cells, and the positive rate of ACE2 in the testes of infertile men was higher than normal, indicating that SARS-CoV-2 may cause or potentiate the reproductive disorders via the ACE2-mediated route of infection ${ }^{[11]}$. In the same study, the proportion of ACE2-positive cells in the testes was found to be more than $1 \%$, which is higher than in the lungs, indicating that testes could serve as a potential high-risk organ of infection ${ }^{[11]}$.

On the other hand, CD147 is a major molecular machinery controlling spermatocyte progression for further differentiation through its dual functionality: promoting migration and cell survival[12]. Additional, CD147 is a ubiquitously expressed protein and is directly involved in spermatogenesis ${ }^{[13]}$ and is expressed at all stages during mouse testicular development, its expression gradually increases in intensity during the differentiation of spermatocytes and spermatids in adult mouse and human testes ${ }^{[12]}$.

Therefore, CD147 receptor recruitment by SARS-CoV-2 may affect fertility through an additional mechanism, and complementation of ACE2 and CD147 pathways in SARS-CoV-2 infection implies a potent susceptibility to testicular infection with possible direct consequences on fertility.

\section{Ciliopathy model to explain the pulmonary pathogenesis and extrapulmonary manifestations in COVID-19}

In human, exist several cellular structures dependent on cilia and flagella, some of these are ${ }^{[14]}$ :

- Mucus propelling cilia: located in respiratory cilia of the upper and lower airways, middle ear

- Water-propelling cilia: located in ependymal cerebral ventricles, testicular ducts, fallopian tubes

- Olfactory cilia: located in the olfactory epithelium

- Sperm flagellum: located in sperm

- Kinocilium: located in the inner ear 
With a first look, we could propose that, among other damages:

- Ciliary alteration of the fallopian tubes could cause decrease in female fertility

- Ciliary alteration of olfactory cilia could cause anosmia or dysosmia

- Ciliary alteration of kinocilium could cause vertigo or peripheral hearing loss

- Ciliary alteration of water- or mucus-propelling cilia could cause complications from tissue local congestion and obstruction ducts, such as: pneumonia and decreased fertility

- Ciliary alteration of sperm flagellum could cause decreased sperm motility and decreased fertility

We want to clarify at this point that cilium can act as mechanic and chemoreceptors, as occurs in the cilia of the inner ear and olfactory receptors, respectively ${ }^{[14]}$.

In ciliopathies, immobility and dyskinesia lead to an absence of mucociliary transport, stasis of respiratory secretions with infections of the upper and lower respiratory tract. The mobility disorder also affects the sperm flagellum and the cilia of the fallopian tube, in these cases infertility is common in men and reduced fertility in women ${ }^{[14]}$.

Clinical presentation is homogeneous (chronic productive cough, rhinorrhea and chronic sinusitis). In addition, there are many ciliary defects acquired by the action of germs or toxins (secondary ciliary dyskinesia). The large number of polypeptides involved in the constitution of the cilium also implies a large number of genes that determine its function, making it difficult to carry out a heterogeneous genetic diagnosis ${ }^{[14]}$.

Genetic heterogeneity with the homogeneous phenotype leads us to consider that any ciliary-flagellar alteration, primary or secondary, will present similar clinical manifestations.

SARS-CoV-2 requires conditions that allow it to reach the lung parenchyma. Thus, the initial alteration of the defence barriers at the level of the ciliated 
respiratory epithelium would represent an adaptive advantage. In addition, the states of bronchial congestion secondary to the alteration of ciliary motility would be a vehicle towards the pulmonary alveoli.

\section{Ciliary alteration in SARS-CoV-2. Transmission electron microscopic and protein interaction studies}

The olfaction of humans and many other species requires the standard structure and function of olfactory cilia, and anosmia turns out to be a common feature of some types of ciliopathies such as Bardet-Biedl syndrome ${ }^{[4]}$.

Viral infection may disrupt the ciliary structure, which abolishes the ciliary localization of olfactory receptors ${ }^{[4]}$.

In 1994, Bjorn Afzelius (who first described primary ciliary dyskinesia) showed that the loss of cilia represented one of the most striking ultrastructural abnormalities in coronavirus-infected cells ${ }^{[15]}$. Two alternative models explain how a cell removes its primary cilium ${ }^{[16]}$. In the amputation model, the entire cilium is acutely eliminated at the ciliary base, and in absorption model, the cilium can be gradually absorbed by the cytoplasm ${ }^{[4]}$. Afzelius reported that cilia retracted into the cell body and found the 9+2 axonemal doublet microtubules inside the cytoplasm, which suggests that viral infection causes deciliation through cilium absorption ${ }^{[4]}$. We consider that the absorption mechanism imposes a greater risk in the face of SARS-CoV-2 infection, since it would imply an internalization of viral particles into the cytoplasm. This could enhance the negative effects of the viral infection in the ciliary ultrastructure.

In the different publications that we reviewed, we only found mention of protein Nsp13 of SARS-CoV-2 in link of interaction with ciliary components of ultrastructure. Nsp13 binds to as many as 12 components in the centrosome, including three in the pericentrosomal region, five in the distal appendage of the centriole, and four in the proximal end of the centriole ${ }^{[4]}$. While we cannot rule out that this interaction is not biologically true, we can argue that Nsp13 may actively localize to the centriole and compete with the endogenous binding partners of the centrosome proteins, abolishing the physiological interactions within the structure ${ }^{[4]}$. 
SARS-CoV-2 shows a similar morphogenetic process as other coronaviruses but causes plaque-like cytopathic effects in human airway epithelial cultures ${ }^{[17]}$. Large areas of disordered cilia were visually confirmed by scanning electron microscopy analysis, indicating that SARS-CoV-2 infection disrupted cilia synchronicity ${ }^{[17]}$.

While there are still no studies of the link between CD147 expression in respiratory ciliary epithelium with SARS-CoV-2 infection, at least it is known that ACE2 receptor protein robustly localizes within the motile cilia of airway epithelial cells, which likely represents the initial or early subcellular site of SARS-CoV-2 viral entry during host respiratory transmission ${ }^{[18]}$. Although the overall intensity of ACE2 expression in the lung is low compared to the kidney, testis, and intestinal villi[18], we know that the SARS-CoV-2 could infect the lung via the CD147-Cyclophilin A-SARS-CoV-2 N route of infection ${ }^{[1]}$.

\section{Human interactome interacts whit SARS-CoV-2 proteome}

In this study, we look for records of human interactome and its interaction with SARS-CoV-2 proteome (high-confidence interactors). We searched for the key interactions that could explain the phenomena of conditioned lung invasion and enhanced by ciliary dyskinesia. This would cause an alteration of the innate immunity of the bronchial tree and congestive states in the lung parenchyma due to difficulty in mucociliary clearance. Other consequences of ciliary and flagellar dyskinesia would be states of infertility and possible cellular defects due to cytoskeleton dysfunction.

We found in the interactome that the CFAP97 human protein (cilia and flagella associated protein 97 ) is capable of interacting with four viral proteins ${ }^{[8]}$. These are:

1. Nucleocapsid phosphoprotein (Nucleoprotein, nuc, ncap, $N$ protein, NCAP_SARS2, GU280_gp10, SARS-CoV-2 N, nucleocapsid, SARSCoV-2 Nucleoprotein)

2. Non-structural protein 16 (2-O-methyltransferase, ORF1ab, R1AB_SARS2, ORF1ab-nsp16, SARS-CoV-2 nsp16, GU280_gp01_nsp16, 2-O-methyltransferase) 
3. Non-structural protein 2 (ORF1ab, R1AB_SARS2, ORF1ab-nsp2, SARSCoV-2 nsp2, GU280_gp01_nsp2)

4. Non-structural protein 7 (ORF1ab, R1AB_SARS2, ORF1ab-nsp7, SARSCoV-2 nsp7, GU280_gp01_nsp7)

About CFAP97 we have given enough arguments to know its determining implication in the human ciliary and flagellar structure (Refer to previous sections.). But, in view of susceptibility of testicular cell infection by SARS-CoV2 and interaction of CFAP97 gene product with these four viral proteins, it is possible the occurrence of sperm motility dysfunction with consequent transient male infertility.

At the same time, SARS-CoV-2 N interact with MAP4 (microtubule-associated protein 4) and SPATS2 (spermatogenesis associated serine-rich 2, P59SCR, SCR59, SPATA10, Nbla00526) human proteins ${ }^{[19]}$, that adds an additional interaction to ciliary-flagellar involvement ${ }^{[20]}$ and male fertility ${ }^{[21]}$, respectively.

Interaction between SARS-CoV-2 N and SPATS2 human protein ${ }^{[19]}$ is capable of enhancing damage to male fertility. Senoo et al. ${ }^{[21]}$ suggested that SPATS2 might play a role in testicular germ cell development in a mice model.

Interaction is SARS-CoV-2 N with MAP4 human protein is of considerable importance ${ }^{[19]}$. MAP4 promotes microtubule assembly, and has been shown to counteract destabilization of interphase microtubule catastrophe promotion. The phosphorylation of this protein affects microtubule properties and cell cycle progression. Multiple alternatively spliced transcription variants encoding different isoforms have been observed ${ }^{[20]}$. MAP4 prevents engagement of excess dynein motors, thereby protecting the system from force imbalance in mitotic spindle positioning process, which is critical to establish the correct cell-division plane ${ }^{[22]}$. MAP4 physically interacts with dynein-dynactin in vivo and inhibits dynein-mediated microtubule sliding in vitro[22]. Considering this, interaction of SARS-CoV-2 N with MAP4 can deregulate one of the most important cellular processes, cell division. We found no evidence that this interaction could produce inhibition of intracellular signal transduction mechanisms or destabilization in 
microtubule-dependent intracellular traffic. However, this is something that definitely requires more investigation.

We also find that PKD2 (polycystic kidney disease 2 protein, polycystin-2, APKD2, PC2, PKD4, Pc-2, TRPP2) is capable of interacting with six SARS-CoV2 proteins ${ }^{[23]}$ :

1. ORF7A (GU280_gp07, NS7A_SARS2, Protein 7a, SARS-CoV-2 ORF7a)

2. ORF7B (GU280_gp08, NS7B_SARS2, SARS-CoV-2 ORF7b)

3. E (emp, env, envelope, E protein, GU280_gp04, VEMP_SARS2, SARSCoV-2 E)

4. M (mem, membrane, M protein, GU280_gp05, VME1_SARS2, SARSCoV-2 M)

5. NSP4 (ORF1ab, R1AB_SARS2, ORF1ab-nsp4, SARS-CoV-2 nsp4, GU280_gp01_nsp4)

6. ORF14 (1Y14_SARS2, GU280_gp14, SARS-CoV-2 ORF14)

PKD2 is a component of a calcium-permeable heteromeric ion channel formed by PKD1 and PKD2 ${ }^{[24]}$ and it functions as a cation channel involved in the mechanosensing of fluid flow through the primary cilium in the renal epithelium ${ }^{25]}$. Together with TRPV4, it forms mechanical and thermosensitive channels in cilium ${ }^{[24]}$.

PKD2 is expressed abundantly in the endoplasmic reticulum membrane and is a high conductance channel that is permeable to divalent cations ${ }^{[26]}$, and has been shown to function at several locations, including primary cilia and basolateral membrane ${ }^{[27]}$. It has been suggested that, PKD2 is essential for its endoplasmic reticulum channel function and links extracellular stimuli to effects on cell growth and intracellular calcium regulation ${ }^{[27]}$. Therefore, it appears that PKD2 interrelates intracellular calcium metabolism with thermosensitivity and mechanosensitivity functions in cilia.

The experimental evidence of ciliary and flagellar alteration in viral infections and, specifically in SARS-CoV-2 infection, alerted us to an underlying molecular alteration without sufficient explanation. Looking for a possible molecular explanation for such studies, we found some protein interactions that must be 
causing a mismatch of the axoneme and, therefore, explain some clinical manifestations of COVID-19 and alert of others. The following table (Table 1) summarizes many of the aspects considered in our review. In Table 1 we can see that ten viral proteins interact with key components in the maintenance of the molecular structure of axoneme.

Table 1. SARS-CoV-2 - human interaction proteins and their link with the cycliar-flagellar structure and the consequent clinical manifestations.

\begin{tabular}{|c|c|c|c|}
\hline Human protein & $\begin{array}{l}\text { SARS-CoV-2 } \\
\text { protein } \\
\text { interaction }\end{array}$ & $\begin{array}{l}\text { Possibly molecular } \\
\text { impaired function }\end{array}$ & $\begin{array}{l}\text { Clinical } \\
\text { consequences }\end{array}$ \\
\hline \multirow{3}{*}{$\begin{array}{l}\text { CFAP97 } \\
\text { (cilia and flagella } \\
\text { associated protein } \\
97 \text { ) }\end{array}$} & $\begin{array}{l}\mathrm{N} \\
\mathrm{nsp} 16\end{array}$ & $\begin{array}{l}\text { General decreased } \\
\text { ciliary and flagellar } \\
\text { movement }\end{array}$ & $\begin{array}{l}\text { Decrease male and } \\
\text { female fertility }\end{array}$ \\
\hline & $\begin{array}{l}\text { nsp2 } \\
\text { nsp7 }\end{array}$ & $\begin{array}{l}\text { Decreased ciliary } \\
\text { movement in fallopian } \\
\text { tubes and decreased } \\
\text { flagellum movement in } \\
\text { sperm }\end{array}$ & $\begin{array}{l}\text { Mucus congestion in } \\
\text { tissues: alteration of } \\
\text { innate bronchial } \\
\text { immunity that favours } \\
\text { pneumonia }\end{array}$ \\
\hline & & $\begin{array}{l}\text { Alteration of ciliated } \\
\text { sensory neurons and } \\
\text { kinocilium }\end{array}$ & $\begin{array}{l}\text { Loss of taste and smell } \\
\text { Vertigo and peripheral } \\
\text { hearing loss }\end{array}$ \\
\hline \multirow[t]{3}{*}{$\begin{array}{l}\text { PKD2 } \\
\text { (polycystic kidney } \\
\text { disease } 2 \text { protein) }\end{array}$} & $\begin{array}{l}\text { ORF7a } \\
\text { ORF7b }\end{array}$ & $\begin{array}{lr}\text { Disruption } & \text { of } \\
\text { mechanical } & \text { and } \\
\text { thermosensitive } & \end{array}$ & $\begin{array}{l}\text { Mucus congestion in } \\
\text { tissues }\end{array}$ \\
\hline & $E$ & channels in cilium & $\begin{array}{l}\text { Disturbance in fluid } \\
\text { transport in kidney }\end{array}$ \\
\hline & $\begin{array}{l}\text { M } \\
\text { nsp4 } \\
\text { ORF14 }\end{array}$ & $\begin{array}{l}\text { Alteration of calcium } \\
\text { permeability in cilium }\end{array}$ & $\begin{array}{l}{ }^{*} \text { Clinical effects due to } \\
\text { disturbances of } \\
\text { intracellular calcium } \\
\text { regulation }\end{array}$ \\
\hline \multirow[t]{4}{*}{$\begin{array}{l}\text { MAP4 } \\
\text { (microtubule- } \\
\text { associated protein } \\
\text { 4) }\end{array}$} & $\mathrm{N}$ & $\begin{array}{l}\text { Destabilization of } \\
\text { interphase microtubule } \\
\text { catastrophe promotion }\end{array}$ & $\begin{array}{l}{ }^{*} \text { Clinical effects due to } \\
\text { alterations in regulation } \\
\text { of processes related to } \\
\text { the cell cycle }\end{array}$ \\
\hline & & $\begin{array}{l}\text { Affects cell } \\
\text { progression }\end{array}$ & \\
\hline & & $\begin{array}{l}\text { Destabilization in } \\
\text { microtubule-dependent } \\
\text { intracellular traffic }\end{array}$ & \\
\hline & & $\begin{array}{l}\text { Inhibition of intracellular } \\
\text { signal transduction } \\
\text { mechanisms }\end{array}$ & \\
\hline
\end{tabular}




\begin{tabular}{|c|c|c|}
\hline $\begin{array}{l}\text { SPATS2 } \\
\text { (spermatogenesis } \\
\text { associated serine- } \\
\text { rich 2) }\end{array}$ & $\begin{array}{l}\text { Alteration of testicular } \\
\text { germ cell development }\end{array}$ & Decrease male fertility \\
\hline
\end{tabular}

\section{Conclusions}

Experiments with the most modern technologies for the study of Virology and Histology have given us a set of evidences on the infection by SARS-CoV-2 in cells with cilia and flagella, a destructive infection of these elements of the membrane. However, they have not been able to give an explanation of the molecular mechanisms underlying the effects they have observed in experimentation. On the other hand, the physical-chemical study of the human interactome in interaction with the SARS-CoV-2 proteome constitute a light that marks the most efficient way to explain the experimental results. With this work, we integrate the experiments in the study of interactions between SARS-CoV-2 proteins with human proteins. Specifically, we refer to ciliary and flagellar dysfunction as the necessary basis to explain the pulmonary and extrapulmonary manifestations that are known to date. In addition, we are alerting about possible episodes of transient infertility in COVID-19 that, due to the limitations of this review, should be verified. We want to open a path for the study of the molecular alterations of the axoneme, from the point of view of the molecular interaction between proteins.

\section{References}

1. Bermejo-Valdés AJ, Cervera-Grau JM (2020) Lighting Up Dark Areas of COVID-19. Ann Case Report 14: 394. DOI: 10.29011/2574-7754.100394. https://www.gavinpublishers.com/articles/case-report/Annals-of-CaseReports/lighting-up-dark-areas-of-covid-19

2. Yan $\mathrm{CH}$, Faraji F, Prajapati DP, Boone CE, DeConde AS. Association of chemosensory dysfunction and COVID-19 in patients presenting with influenza-like symptoms. Int Forum Allergy Rhinol. 2020;10(7):806-813. doi:10.1002/alr.22579.

https://www.ncbi.nlm.nih.gov/pmc/articles/PMC7262089/ 
3. Centers for Disease Control and Prevention (2020) Symptoms of Coronavirus. Available from: https://www.cdc.gov/coronavirus/2019ncov/symptoms-testing/symptoms.html

4. Li W, Li M, Ou G. COVID-19, cilia, and smell [published online ahead of print, 2020 Jul 21]. FEBS J. 2020;287(17):3672-3676. doi:10.1111/febs.15491. https://www.ncbi.nlm.nih.gov/pmc/articles/PMC7426555/

5. Soulavie F, Piepenbrock D, Thomas J, Vieillard J, Duteyrat JL, Cortier E, Laurençon A, Göpfert MC, Durand B. hemingway is required for sperm flagella assembly and ciliary motility in Drosophila. Mol Biol Cell. 2014 Apr;25(8):1276-86. doi: 10.1091/mbc.E13-10-0616. Epub 2014 Feb 19. PMID: 24554765;

PMCID:

PMC3982993. https://pubmed.ncbi.nlm.nih.gov/24554765/

6. Marcus A. Mall. Role of Cilia, Mucus, and Airway Surface Liquid in Mucociliary Dysfunction: Lessons from Mouse Models. Journal of Aerosol Medicine and Pulmonary Drug Delivery 2008 21:1, 13-24. https://www.liebertpub.com/doi/abs/10.1089/jamp.2007.0659

7. Sironen A, Shoemark A, Patel M, Loebinger MR, Mitchison HM. Sperm defects in primary ciliary dyskinesia and related causes of male infertility. Cell Mol Life Sci. 2020 Jun;77(11):2029-2048. doi: 10.1007/s00018-019-033897. Epub 2019 Nov 28. PMID: 31781811; PMCID: PMC7256033. https://pubmed.ncbi.nlm.nih.gov/31781811/

8. Estelle M.N. Laurent, Yorgos Sofianatos, Anastassia Komarova, Jean-Pascal Gimeno, Payman Samavarchi Tehrani, Dae-Kyum Kim, et al . SARS-CoV-2 proteins BiolD analysis suggests new pathogenic mechanisms (2020). bioRxiv. https://doi.org/10.1101/2020.08.28.272955. The interactomes for CFAP97 are available from: BioGRID COVID-19 Coronavirus Curation Project. https://thebiogrid.org/121639/summary/homo-sapiens/cfap97.html

9. Oura S, Kazi S, Savolainen A, Nozawa K, Castañeda J, Yu Z, Miyata H, Matzuk RM, Hansen JN, Wachten D, Matzuk MM, Prunskaite-Hyyryläinen R. Cfap97d1 is important for flagellar axoneme maintenance and male mouse fertility. PLoS Genet. 2020 Aug 12;16(8):e1008954. doi: 10.1371/journal.pgen.1008954. PMID: 32785227; PMCID: PMC7444823. https://pubmed.ncbi.nlm.nih.gov/32785227/ 
10. Zur Lage P, Newton FG, Jarman AP. Survey of the Ciliary Motility Machinery of Drosophila Sperm and Ciliated Mechanosensory Neurons Reveals Unexpected Cell-Type Specific Variations: A Model for Motile Ciliopathies. Front Genet. 2019 Feb 1;10:24. doi: 10.3389/fgene.2019.00024. PMID: 30774648;

PMCID:

PMC6367277.

https://pubmed.ncbi.nlm.nih.gov/30774648/

11. Shen, $Q$, Xiao, X, Aierken, A, et al. The ACE2 expression in Sertoli cells and germ cells may cause male reproductive disorder after SARS-CoV-2 infection. J Cell Mol Med. 2020; 24: 9472- 9477. https://doi.org/10.1111/jcmm.15541

12. Hao Chen, Kin Lam Fok, Xiaohua Jiang \& Hsiao Chang Chan (2012) New insights into germ cell migration and survival/apoptosis in spermatogenesis, Spermatogenesis, 2:4, 264-272, DOI: 10.4161/spmg.22014. https://www.tandfonline.com/doi/full/10.4161/spmg.22014

13. Yurchenko, V., Constant, S. and Bukrinsky, M. (2006), Dealing with the family: CD147 interactions with cyclophilins. Immunology, 117: 301-309. https://doi.org/10.1111/j.1365-2567.2005.02316.x

14. Miguel Armengot Carceller, Manuel Mata Roig, Xavier Milara Payá, Julio Cortijo Gimeno, Discinesia ciliar primaria. Ciliopatías, Acta Otorrinolaringológica Española, Volume 61, Issue 2, 2010, Pages 149-159, ISSN 0001-6519. https://doi.org/10.1016/j.otorri.2009.01.013

15. Afzelius BA. Ultrastructure of human nasal epithelium during an episode of coronavirus infection. Virchows Arch. 1994;424(3):295-300. doi: 10.1007/BF00194614. PMID: 8186894; PMCID: PMC7087561. https://pubmed.ncbi.nlm.nih.gov/8186894/

16. Mirvis M, Siemers KA, Nelson WJ, Stearns TP. Primary cilium loss in mammalian cells occurs predominantly by whole-cilium shedding. PLoS Biol. 2019 Jul 17;17(7):e3000381. doi: 10.1371/journal.pbio.3000381. PMID: 31314751 ;

PMCID:

PMC6699714.

https://pubmed.ncbi.nlm.nih.gov/31314751/

17.Zhu, N., Wang, W., Liu, Z. et al. Morphogenesis and cytopathic effect of SARS-CoV-2 infection in human airway epithelial cells. Nat Commun 11, 3910 (2020). https://doi.org/10.1038/s41467-020-17796-z 
18. Lee, I.T., Nakayama, T., Wu, CT. et al. ACE2 localizes to the respiratory cilia and is not increased by ACE inhibitors or ARBs. Nat Commun 11, 5453 (2020). https://doi.org/10.1038/s41467-020-19145-6

19. Estelle M.N. Laurent, Yorgos Sofianatos, Anastassia Komarova, Jean-Pascal Gimeno, Payman Samavarchi Tehrani, Dae-Kyum Kim, et al . SARS-CoV-2 proteins BiolD analysis suggests new pathogenic mechanisms (2020). bioRxiv. https://doi.org/10.1101/2020.08.28.272955. The interactomes for SARS-CoV-2 $\mathrm{N}$ are available from: BioGRID COVID-19 Coronavirus Curation Project. https://thebiogrid.org/4383847/summary/severe-acuterespiratory-syndrome-coronavirus-2/n.html

20. Entrez Gene: MAP4 microtubule-associated protein 4. https://www.ncbi.nlm.nih.gov/gene?Db=gene\&Cmd=ShowDetailView\&Term ToSearch $=4134$

21. Makoto Senoo, Shigetaka Hoshino, Naoko Mochida, Yasuko Matsumura, Sonoko Habu. Identification of a Novel Protein p59scr, Which Is Expressed at Specific Stages of Mouse Spermatogenesis. Biochemical and Biophysical Research Communications. Volume 292, Issue 4. 2002. Pages 992-998. ISSN 0006-291X. https://doi.org/10.1006/bbrc.2002.6769. http://www.sciencedirect.com/science/article/pii/S0006291X02967690

22. Samora, C., Mogessie, B., Conway, L. et al. MAP4 and CLASP1 operate as a safety mechanism to maintain a stable spindle position in mitosis. Nat Cell Biol 13, 1040-1050 (2011). https://doi.org/10.1038/ncb2297

23. Estelle M.N. Laurent, Yorgos Sofianatos, Anastassia Komarova, Jean-Pascal Gimeno, Payman Samavarchi Tehrani, Dae-Kyum Kim, et al . SARS-CoV-2 proteins BiolD analysis suggests new pathogenic mechanisms (2020). bioRxiv. https://doi.org/10.1101/2020.08.28.272955. The interactomes for PKD2 are available from: BioGRID COVID-19 Coronavirus Curation Project.https://thebiogrid.org/111328

24. Kim S, Nie H, Nesin V, Tran U, Outeda P, Bai CX, Keeling J, Maskey D, Watnick T, Wessely O, Tsiokas L. The polycystin complex mediates Wnt/Ca(2+) signalling. Nat Cell Biol. 2016 Jul;18(7):752-764. doi: 10.1038/ncb3363. Epub 2016 May 23. PMID: 27214281; PMCID: PMC4925210. https://pubmed.ncbi.nlm.nih.gov/27214281/ 
25. Köttgen M, Buchholz B, Garcia-Gonzalez MA, Kotsis F, Fu X, Doerken M, Boehlke C, Steffl D, Tauber R, Wegierski T, Nitschke R, Suzuki M, KramerZucker A, Germino GG, Watnick T, Prenen J, Nilius B, Kuehn EW, Walz G. TRPP2 and TRPV4 form a polymodal sensory channel complex. J Cell Biol. 2008 Aug 11;182(3):437-47. doi: 10.1083/jcb.200805124. PMID: 18695040; PMCID:

PMC2500130. https://www.ncbi.nlm.nih.gov/pmc/articles/PMC2500130/

26. Koulen, P., Cai, Y., Geng, L. et al. Polycystin-2 is an intracellular calcium release channel. Nat Cell Biol 4, 191-197 (2002). https://doi.org/10.1038/ncb754

27. Streets AJ, Needham AJ, Gill SK, Ong AC. Protein kinase D-mediated phosphorylation of polycystin-2 (TRPP2) is essential for its effects on cell growth and calcium channel activity. Mol Biol Cell. 2010 Nov 15;21(22):385365. doi: 10.1091/mbc.E10-04-0377. Epub 2010 Sep 29. PMID: 20881056; PMCID: PMC2982124. https://pubmed.ncbi.nlm.nih.gov/20881056/ 